\title{
Radioembolization Versus Bland Embolization for Hepatic Metastases from Small Intestinal Neuroendocrine Tumors: Short- Term Results of a Randomized Clinical Trial
}

\author{
Anna-Karin Elf ${ }^{1} \cdot$ Mats Andersson $^{2} \cdot$ Olof Henrikson $^{2} \cdot$ Oscar Jalnefjord $^{3}$ • \\ Maria Ljungberg $^{3} \cdot$ Johanna Svensson $^{4} \cdot$ Bo Wängberg $^{1} \cdot$ Viktor Johanson $^{1}$
}

Published online: 22 November 2017

(C) The Author(s) 2017. This article is an open access publication

\begin{abstract}
Background Radioembolization (RE) with intra-arterial administration of ${ }^{90} \mathrm{Y}$ microspheres is a promising technique for the treatment of liver metastases from small intestinal neuroendocrine tumors (SI-NET) not amenable to surgery or local ablation. However, studies comparing RE to other loco-regional therapies are lacking. The aim of this randomized study was to compare the therapeutic response and safety after RE and bland hepatic arterial embolization (HAE), and to investigate early therapy-induced changes with diffusion-weighted MRI (DWI-MRI). Methods Eleven patients were included in a prospective randomized controlled pilot study, six assigned to RE and five to HAE. Response according to RECIST 1.1 using MRI or CT at 3 and 6 months post-treatment was recorded as well as changes in DWI-MRI parameters after 1 month. Data on biochemical tumor response, toxicity, and side effects were also collected.

Results Three months after treatment, all patients in the HAE group showed partial response according to RECIST while none in the RE group did $(p=0.0022)$. After 6 months, the response rates were $4 / 5(80 \%)$ and $2 / 6(33 \%)$ in the HAE and RE groups, respectively (NS). DWI-MRI metrics could not predict RECIST response, but lower pretreatment $\mathrm{ADC}_{(120-800)}$ and larger $\mathrm{ADC}_{(0-800)}$ increase at 1 month were related to larger decrease in tumor diameter when all tumors were counted.

Conclusion HAE resulted in significantly higher RECIST response after 3 months, but no difference compared to RE remained after 6 months. These preliminary findings indicate that HAE remains a safe option for the treatment of liver metastases from SI-NET, and further studies are needed to establish the role of RE and the predictive value of MR-DWI.
\end{abstract}

Electronic supplementary material The online version of this article (https://doi.org/10.1007/s00268-017-4324-9) contains supplementary material, which is available to authorized users.

Anna-Karin Elf and Mats Andersson have contributed equally to the completion of this manuscript.

Anna-Karin Elf

anna-karin.elf@vgregion.se

1 Department of Surgery, Sahlgrenska University Hospital and Sahlgrenska Academy, University of Gothenburg, Gothenburg, Sweden

\section{Introduction}

The primary tumors of small intestinal neuroendocrine tumors (SI-NETs) can almost always be radically resected by surgery, often together with the regional lymph node metastases. Hepatic metastases are common, and surgical

2 Department of Radiology, Sahlgrenska University Hospital and Sahlgrenska Academy, University of Gothenburg, Gothenburg, Sweden

3 Department of Radiation Physics, Sahlgrenska University Hospital and Sahlgrenska Academy, University of Gothenburg, Gothenburg, Sweden 
resection is suitable only in a minority of cases calling for other loco-regional treatment alternatives [1]. Local ablative therapies using radiofrequency or microwaves are an option if the metastases are few, small, and suitably localized. In other cases, loco-regional endovascular therapies, such as hepatic arterial embolization (HAE) or chemoembolization (TACE), are considered. The mainstay for treating widespread liver metastases at our unit has been HAE with polyvinyl alcohol (PVA) particles [2]. However, the treatment is often associated with side effects due to liver ischemia, and recurrence may occur. Recently, radioembolization ( $\mathrm{RE}$ ), involving transcatheter arterial delivery of $20-60 \mu \mathrm{m}$ microspheres containing ${ }^{90} \mathrm{Y}$ radioisotope into the tumor microvasculature, has emerged as a promising tool in the management of hepatic metastases from NET. RE is considered to have less acute and subacute toxicity than HAE, as it avoids liver ischemia. However, studies documenting the safety and efficacy of RE largely represent retrospective cohort series [3-9], while prospective studies comparing RE to other loco-regional therapies such as HAE are lacking.

Currently accepted guidelines for assessing treatment response in solid tumors include response evaluation criteria in solid tumors (RECIST) $[10,11]$. However, these morphological criteria may not be conclusive until several months following therapy. Earlier evaluation of response to therapy would allow alternative therapy sooner, with optimized management of the individual patient. Diffusionweighted imaging (DWI) is a functional MR imaging method that provides insight into the tumor microenvironment and can potentially be used as an early surrogate biomarker for tumor response [12, 13].

Here, we present the preliminary results of a randomized phase II study where the primary end point was to compare the treatment response, according to RECIST, of hepatic metastases at 3 months after RE or HAE. Secondary aims were to study the radiological response at 6 months, the biochemical response, and toxicity, and to evaluate the usefulness of early changes in DWI parameters in predicting later treatment response.

\section{Materials and methods}

\section{Patients}

Inclusion criteria were multiple SI-NET liver metastases, grade 1 or 2 , not accessible to curative resection or ablation, and elevated serum chromogranin $\mathrm{A}(\mathrm{CgA})$ and/or

4 Department of Oncology, Sahlgrenska University Hospital and Sahlgrenska Academy, University of Gothenburg, Gothenburg, Sweden
$24 \mathrm{~h}$ urinary 5-HIAA excretion (dU-5HIAA). All patients were under somatostatin analogue (SSA) treatment, and primary surgery with removal of all extrahepatic tumors had been performed in all patients.

Exclusion criteria were remaining extrahepatic metastases, previous loco-regional or systemic anti-tumoral treatment (except SSA), impaired liver function or tumor volume exceeding $50 \%$ of total liver volume.

Before treatments, all patients but one were investigated with DWI-MRI. The remaining patient could not undergo MRI due to cardiac pacemaker and was evaluated with CT. DWI-MRI was repeated 1 month after treatment followed by response evaluation with MRI or CT according to RECIST 1.1 at 3 and 6 months.

The study was approved by the Ethical Review Board of Gothenburg University.

\section{RE and HAE procedures}

Radioembolization was performed with bilobar infusion in a standard manner in all patients [9]. Protective coil embolization was used when necessary to prevent nontarget embolization. The administered activity of ${ }^{90} \mathrm{Y}$ resin microspheres (SIR-spheres ${ }^{\mathrm{TM}}$ ) was calculated using the partition model [14].

HAE was performed by infusion of PVA particles (45-150 $\mu \mathrm{m})$ into the right or left hepatic artery until stasis was achieved. The right liver lobe was treated first, embolizing the remaining left lobe at a second session, about 6 weeks later. No other anti-tumoral therapy was started during the study period.

\section{Magnetic resonance imaging}

The MRI measurements were performed on a Gyroscan Achieva dStream 3 T Release 5.1.7 (Philips Medical Systems, Eindhoven, the Netherlands). The examination included T2W and T1W scans, and a DWI scan with multiple $b$ values $\left(0,120,350,575,800 \mathrm{~s} / \mathrm{mm}^{2}\right)$. At baseline and at the 3 and 6 months follow-up examinations, a T1-weighted dynamic contrast-enhanced sequence in the late arterial and portal venous phases was added to the protocol (for details see Electronic Supplementary Material).

\section{Image analysis}

Up to five liver metastases, with lesion diameters larger than $1 \mathrm{~cm}$, were analyzed per patient. Multiple free-hand regions of interest (ROIs) were drawn to encompass the whole volume of the metastases on DWI-MRI images. The relative $\mathrm{ADC}$ values at 1 month, compared to pretreatment baseline values, were calculated. As a control, a circular ROI of minimum $120 \mathrm{~mm}^{2}$ was placed on normal- 
appearing splenic parenchyma (for details see Electronic Supplementary Material).

\section{Assessment of therapeutic response}

Response evaluation was performed by MRI or CT at 3 and 6 months after treatment, according to the RECIST 1.1 guidelines [11]. For every patient, two target lesions, which appeared best measurable and representative of the patient's overall tumor load at the baseline MRI/CT, were chosen in accordance with RECIST criteria using the "Tumor Tracking" module at the IntelliSpace-Portal workstation.

Biochemical tumor markers $\mathrm{CgA}$ in serum and dU5HIAA were measured at 3 and 6 months after treatment.

Therapeutic response at 3 and 6 months after treatment was also determined on a lesion-by-lesion basis by evaluating changes in size of a total of 39 tumors. The measurements of the lesion diameters (LD = longest diameter) were averaged across all MR sequences. The relative tumor size at different times after treatment was calculated by comparing it with pretreatment baseline values (for details see Electronic Supplementary Material).

\section{Toxicity}

Toxicity was assessed weekly during the first month after treatment by measuring hemoglobin, white blood count, and platelets. At the same time points, liver-specific toxicity was assessed by measuring ASAT, ALAT, ALP, and bilirubin. Blood sampling was repeated at 3 and 6 months after treatments.

\section{Statistical analysis}

Response rates according to RECIST1.1 were estimated with binomial proportions and compared by Fisher's exact test using the MedCalc statistics package (MedCalc Software, Ostend, Belgium). Linear mixed models were used to test for difference between changes in ADC parameters and for linear correlation between ADC parameters and change in size following treatment using MATLAB 2016b (MathWorks Inc, Natick, MA, USA). Mann-Whitney $U$ test or Wilcoxon signed-rank test were used to compare differences in continuous variables. A $p$ value of $<0.05$ was considered statistically significant.

\section{Results}

Between January 2014 and September 2016, 11 patients with irresectable liver metastases from SI-NET, not suitable for local ablative treatments, were randomly assigned to RE $(n=6)$ or HAE $(n=5)$. Median age at enrollment was 67 years, and three patients were men (Table 1). All patients had $>5$ metastases, and a total of 36 lesions, which could be consistently assessed throughout all MRI examinations, were analyzed on a lesion-by-lesion basis. A median activity of 1.4 (range $1.1-1.8$ ) $\mathrm{GBq}$ of ${ }^{90} \mathrm{Y}$ was given to patients receiving $\mathrm{RE}$. The first radiological response evaluation, DWI-MRI, was performed after a median time of 32 days (range 22-55 days) after RE or the first HAE treatment (the right lobe). The 2nd and 3rd evaluations were performed after a median time of 90 and 195 days, respectively (range 84-118 and 166-251 days), after treatment. However, one of the patients, with a pacemaker, was assessed with CT only, and another patient

Table 1 Baseline data: Baseline clinical and tumor characteristics of the study population randomized to RE or HAE

\begin{tabular}{|c|c|c|c|c|}
\hline & $\begin{array}{l}\text { All patients } \\
(n=11)\end{array}$ & $\begin{array}{l}\text { Patients receiving } \\
\mathrm{RE}(n=6)\end{array}$ & $\begin{array}{l}\text { Patients receiving } \\
\text { HAE }(n=5)\end{array}$ & $p$ value \\
\hline Age, years, median (range) & $67(40-79)$ & $66.5(40-79)$ & $67(51-79)$ & $0.65 *$ \\
\hline Male sex & 3 & 2 & 1 & $1.00 * *$ \\
\hline Number of lesions analyzed, median (range) & $4(1-5)$ & $5(2-5)$ & $3(1-5)$ & $0.11 *$ \\
\hline Median LD, mm (range) & $20.3(13-55)$ & $20.3(13-50)$ & $19.9(13-55)$ & $0.92 *$ \\
\hline Median sum of LD of metastases analyzed, $\mathrm{mm}$ (range) & $77(30-170)$ & $89(35-170)$ & $74(30-170)$ & $0.27 *$ \\
\hline Median baseline $\mathrm{ADC}_{(120-800)}, 10^{-3} \mathrm{~mm}^{2} / \mathrm{s}$ (range) & $0.73(0.5-1.3)$ & $0.78(0.5-1.3)$ & $0.68(0.5-1.0)$ & $0.15^{*}$ \\
\hline Primary tumor, grade $1(\mathrm{Ki}-67<2 \%)(n)$ & 7 & 5 & 2 & $0.24 * *$ \\
\hline Primary tumor, grade $2(\mathrm{Ki}-673-20 \%)(n)$ & 4 & 1 & 3 & $0.24 * *$ \\
\hline Median dU-5HIAA ( $\mu \mathrm{mol} / 24 \mathrm{~h})$ (range) & $110(21-270)$ & $97(54-130)$ & $110(21-270)$ & $0.93 *$ \\
\hline Median $\mathrm{CgA}(\mu \mathrm{g} / \mathrm{L})$ (range) & $231(81-1890)$ & $162(81-470)$ & $384(115-1890)$ & $0.36 *$ \\
\hline
\end{tabular}

* Mann-Whitney $U$ test

** Fisher's exact test 
treated with HAE failed to undergo DWI-MRI at the 1-month time point.

\section{RECIST response}

At 3 months after treatment, no responders were seen in the RE group while all patients in the HAE group showed partial response $(\mathrm{PR})(p=0.0022)$. After 6 months, two patients in the RE group showed PR while one patient in the HAE group had progressed compared to nadir at 3 months, resulting in no remaining significant difference between the groups (Fisher's exact test, $p=0.24$ ) (Fig. 1).

\section{Biochemical response}

Biochemical markers, CgA and 5HIAA, decreased in most patients after 3 and 6 months. The data were rather divergent, and there were no statistically significant differences between the treatment groups. The median reduction in $\mathrm{CgA}$ level after 3 months was $52 \%$ in the HAE group and $29 \%$ in the RE group. Corresponding values after 6 months were 47 and 44\%, respectively (Fig. 2). The median reduction in 5HIAA excretion after 3 months was $43 \%$ in the HAE group and $25 \%$ in the RE group. Corresponding values after 6 months were 36 and $43 \%$, respectively (Fig. 3).

\section{Tumor size response and correlation with DWI assessment}

The median pretreatment LD was $20 \mathrm{~mm}$ (range 13-55 mm) when evaluating all 36 tumors. In the 10 patients examined with MRI, pretreatment $\mathrm{ADC}_{(120-800)}$ values were significantly negatively correlated with treatment response (decreasing LD) at 6 months $(k=45$, $p<0.01)$. The mean increase in $\mathrm{ADC}_{(120-800)}$ at 1 month was $40 \%$ after HAE and $11 \%$ after RE, although the difference between treatments was not statistically significant ( $p=0.08)$. However, there was a statistically significant correlation between the relative increase in the $\mathrm{ADC}_{(0-800)}$ values at 1 month and treatment response (decreasing LD) at 3 months $(k=-0.2, p<0.05)$ (Fig. 4). After 3 and 6 months, the relative (compared to baseline) median LD was significantly smaller in metastases treated with HAE than in patients treated with RE, 45 versus $89 \%$ $(p<0.001)$ and 39 versus $81 \%(p<0.001)$, respectively (Fig. 5).

No difference in the median $\mathrm{ADC}_{(0-800)}$ or $\mathrm{ADC}_{(120-800)}$ values measured in the spleen before and after treatment was found (data not shown).

\section{Toxicity}

Overall toxicity from both treatments was low, and blood cell count at 6 months did not differ from baseline. Liver-

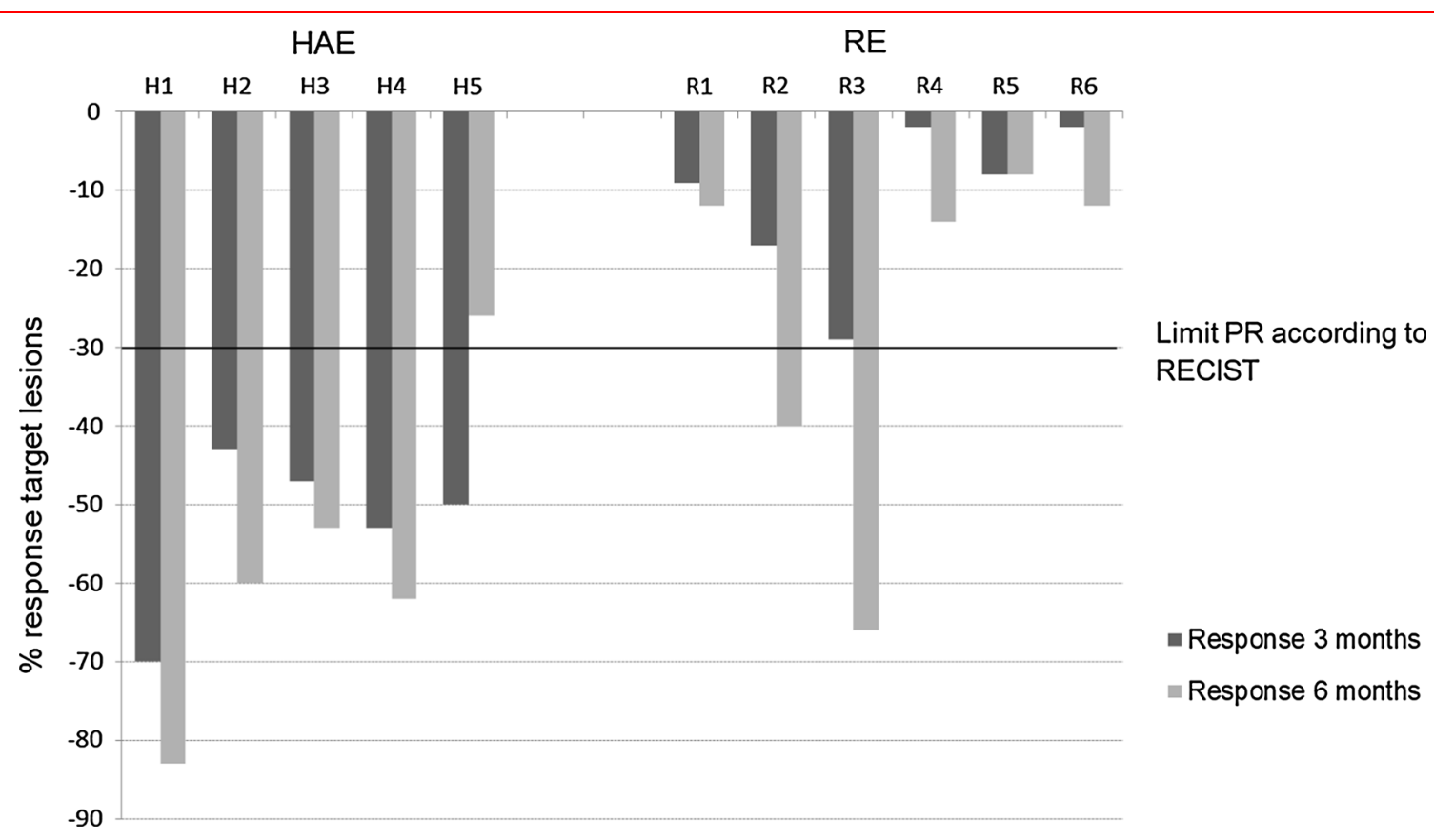

Fig. 1 RECIST response in target lesions in the treated liver at 3 months (dark gray staples) and 6 months (light gray staples) post-treatment. Solid black line indicates threshold for partial response (PR). At 3 months, no responders were seen in the RE group while all patients in the HAE group showed PR $(p=0.0022)$ 


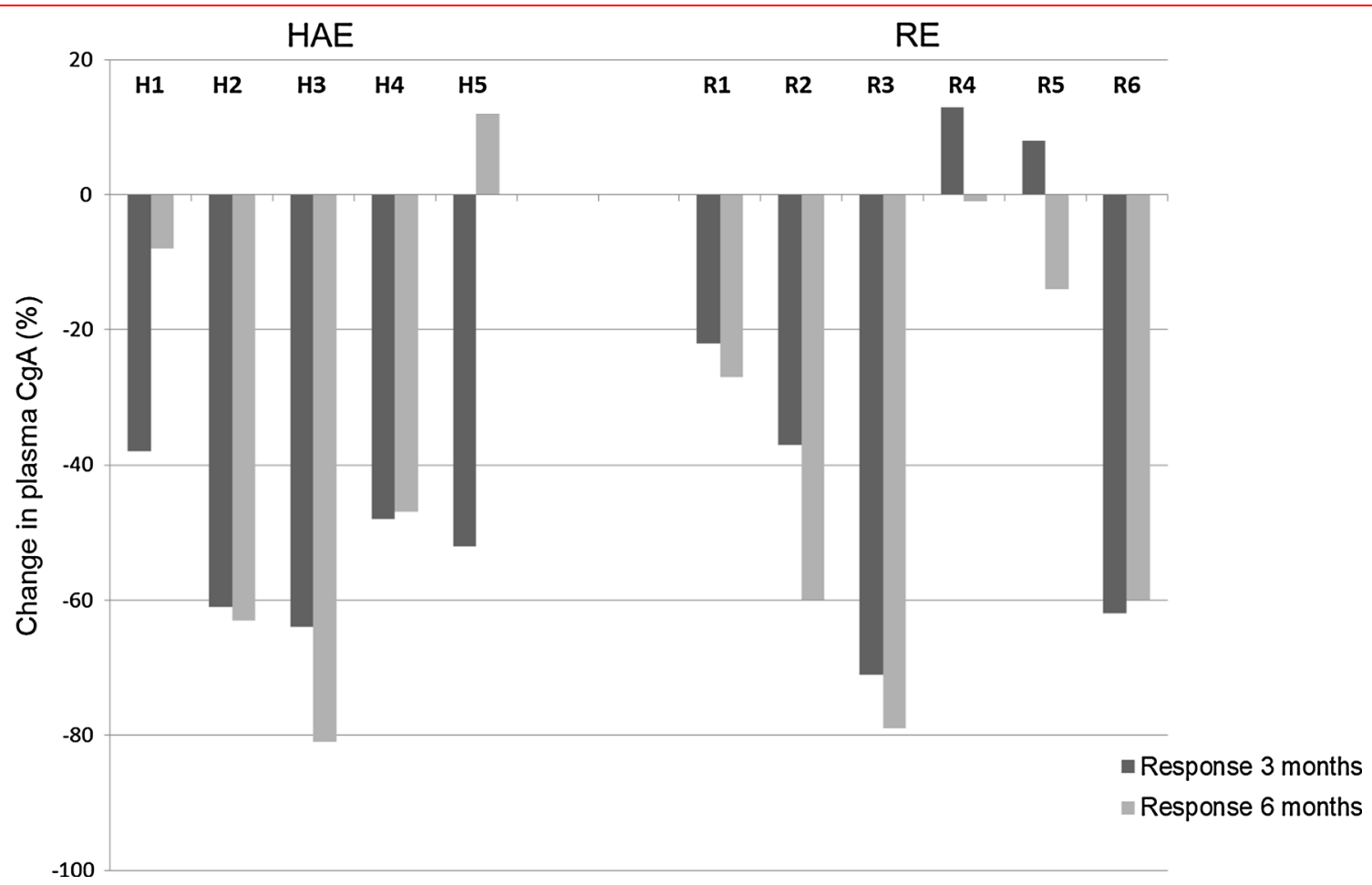

Fig. 2 Relative change in CgA levels compared to baseline values, at 3 months (dark gray staples) and at 6 months (light gray staples) after treatment with HAE or RE. The median decrease in CgA levels at 3 months was 52\% in the HAE group and $29 \%$ in the RE group, and 47 and $44 \%$, respectively, at 6 months (NS, $p=0.42$ and 0.66 , respectively)

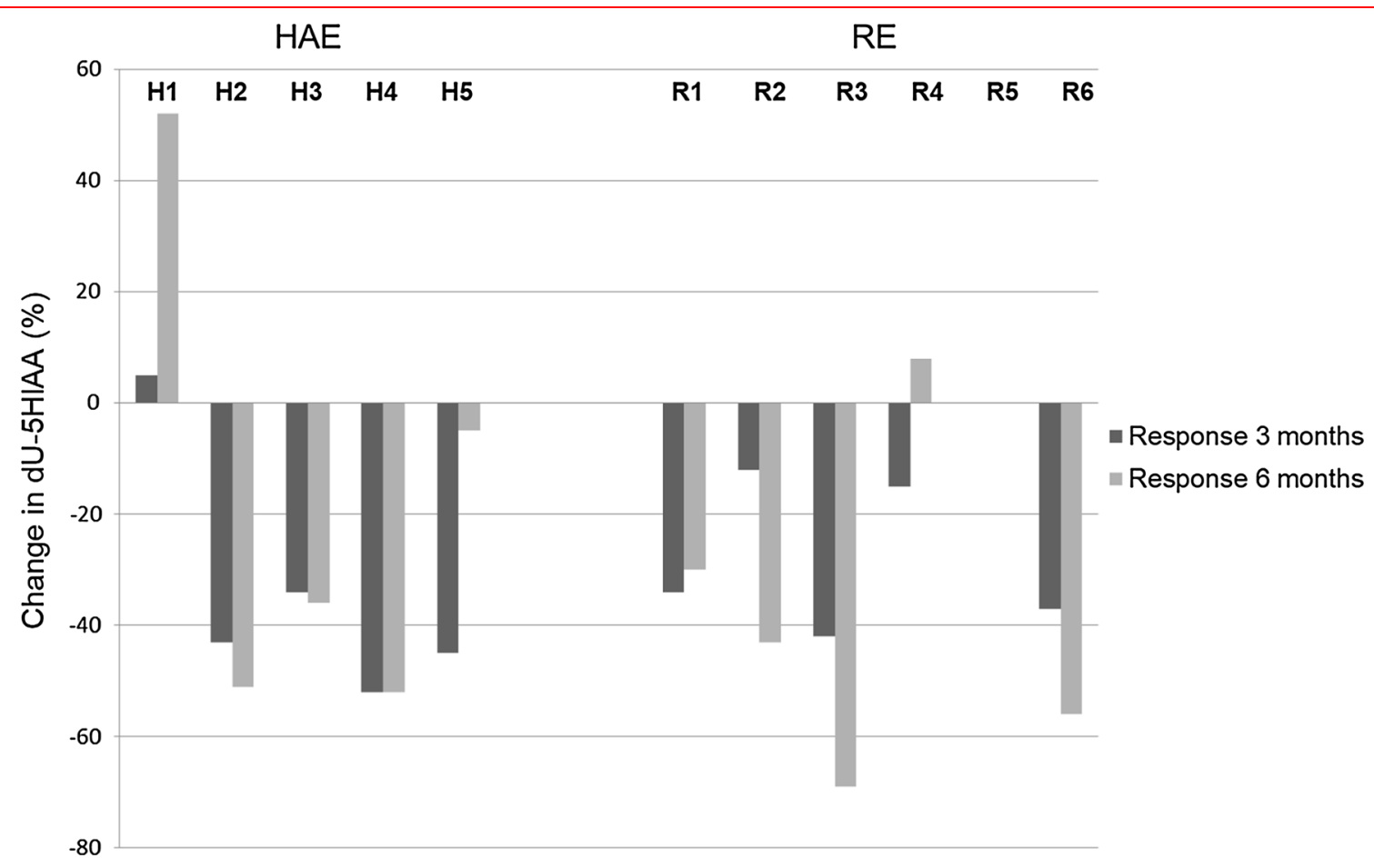

Fig. 3 Relative change in dU-5HIAA compared to baseline values, at 3 months (dark gray staples) and at 6 months (light gray staples) after treatment with HAE or RE. The median decrease in dU-5HIAA at 3 months was $43 \%$ in the HAE group and $25 \%$ in the RE group, and 36 and $43 \%$, respectively, at 6 months (NS, $p>0.05$ ). Patient R5 showed no change in dU-5HIAA values at all time points, hence no visible bars in the figure 


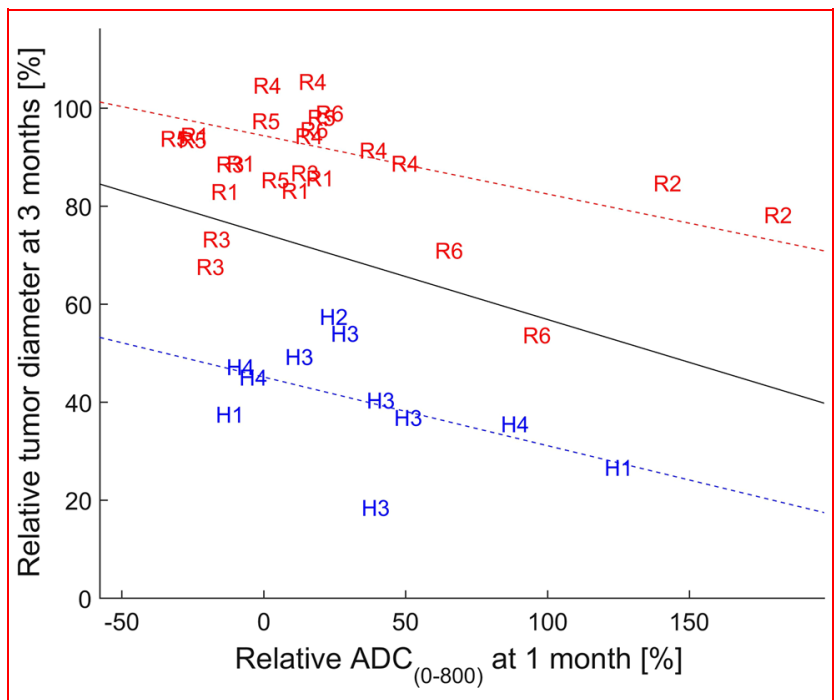

Fig. 4 Correlation between $\mathrm{ADC}_{(0-800)}$ at 1 month and decrease in LD at 3 months $(k=-0.2, p<0.05)$ in individual tumors in patients treated with RE $(\mathrm{R})$ and $\operatorname{HAE}(\mathrm{H})$, respectively. Values are given as percent of pretreatment value. The blue, red, and black lines show the regression line for HAE patients, RE patients, and all patients, respectively

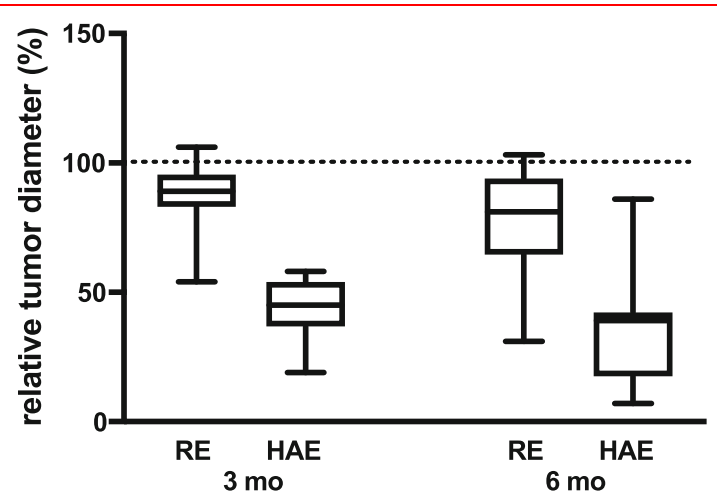

Fig. 5 Relative individual tumor diameters after treatment. At 3 months after treatment, the relative (compared to baseline) median tumor diameter in measured lesions was significantly smaller after HAE, $45 \%$ (range 19-58\%), compared to RE, $89 \%$ (range $54-106 \%),(p<0.001)$. The difference remained after 6 months, when the relative tumor diameter was 39\% (range $7-86 \%$ ) after HAE and $81 \%$ (range $31-103 \%)$ after RE $(p<0.001)$

specific evaluation showed that RE patients had slightly increased median levels of alkaline phosphatase compared to HAE patients at 6 months $(2.8 \mu \mathrm{kat} / \mathrm{L}$, range $1.6-15.5$ vs. $1.45 \mu \mathrm{kat} / \mathrm{L}$, range $1.0-2.1, p<0.05$ ), but no significant differences in liver enzymes or bilirubin values were found.

One patient in the HAE group developed post-treatment cholecystitis, which caused a prolonged hospital stay of 10 days. Median hospital stay after HAE was 4 days (range
4-10 days), which was significantly $(p=0.024)$ longer than after RE (median 2 days, range $2-6$ days).

\section{Discussion}

For SI-NET patients with metastases that cannot be resected by surgery, other treatment options are needed. Systemic radiotherapy with radiolabeled somatostatin analogues (PRRT) has recently proved to be superior to treatment with somatostatin analogues alone [15]. But accumulated radiotoxicity is a concern, and repeated fulldose PRRT can rarely be performed. When the metastatic burden is limited to the liver, liver-directed therapy is preferable. At least HAE can be performed repeatedly, and neither HAE nor RE prevents future use of PRRT, if needed [16].

This is the first randomized prospective study that compares HAE and RE for the treatment of hepatic SI-NET metastases. Response evaluation according to RECIST utilizes the longest lesion diameter (LD) and specified threshold diameter changes. However, the appearance and size of liver metastases from NET may vary between MR sequences and phases of contrast administration [17]. It is currently somewhat unclear which sequence is the best for measuring these lesions [18]. Peri-tumoral edema on T2W imaging and peri-tumoral enhancement on dynamic contrast imaging may cause overestimation of the diameter measured. We hypothesized that averaging LD across all imaging sequences at one time point would compensate for these differences, optimizing the conditions for accurate response evaluation.

In this study, the RECIST response rate after radioembolization for metastatic NET was 33\% at 6 months, which compares well with other studies that have shown a response rate between 22 and 63\% [4-6, 19-22]. We noted that compared to HAE, the response after RE was delayed and that the target lesions continued to decrease in size from 3 to 6 months post-treatment. At 6 months, no significant difference in the RECIST response remained. A similar trend in the response pattern of the biochemical tumor markers $\mathrm{CgA}$ and 5HIAA was noted. Such a delayed treatment response has also been observed by Fidelman et al. [23] who reported a median time to maximum response of 11 months in a study using ${ }^{90} \mathrm{Y}$ glass microspheres for the treatment of metastatic NET.

$\mathrm{ADC}_{(120-800)}$ primarily reflects diffusion, and both animal models and clinical studies have shown that lower ADC values in tumors indicate high viability [12] and a relative lack of necrosis, facilitating the intratumoral delivery of therapeutic agents $[24,25]$. The finding that lower pretreatment $\mathrm{ADC}_{(120-800)}$ was related to improved response (larger decrease of LD at 6 months) represents a 
well-known association between pretreatment ADC and response that has been reported for NET as well as for other tumor entities [26-28].

While HAE resulted in a significant increase in $\mathrm{ADC}_{(120-800)}$ at 1 month, $\mathrm{RE}$ did not. The reason for this is not clear, although the radiotoxic effect certainly is delayed compared to the more immediate ischemic effect of bland embolization. Another study of RE found no early ADC increase in responding colorectal liver metastases, which they associated with cellular edema [29]. Signs of an inflammatory response with peri-tumoral edema after RE have also been described on CT imaging [30]. However, it cannot be ruled out that the lack of a significant increase in diffusion at 1 month after RE was due to inadequate antitumor effect or suboptimal timing of the DWI measurement.

DWI analysis at 1 month after treatment could not predict the RECIST response in an individual patient in this small study. A larger increase in $\mathrm{ADC}_{(0-800)}$ was, however, related to improved response (larger decrease of LD at 3 months) when analyzing all measured lesions. Since $\mathrm{ADC}_{0-800}$, as opposed to $\mathrm{ADC}_{120-800}$, is affected by both diffusion and pseudodiffusion caused by microscopic circulation, this result may indicate that changes in microperfusion after embolization are involved in the therapeutic process. Although there are contradictory results in the literature [31], this finding is in accordance with the suggestion of Kukuk et al. [26] that there is an increase in microperfusion secondary to a decrease in interstitial fluid pressure (IFP) in responding NET metastases.

This study has several limitations. Being an interim analysis of a pilot study, the study population is small, which limits the conclusions that can be drawn. The effect of inter-observer variation in the ROI positioning was not investigated, but it has been shown that this variation is less important in whole-volume measurements, as in our study [32].

Strength of the study is the prospective randomized design. To our knowledge, this is the first randomized trial comparing outcome after RE versus HAE in patients with metastatic NET.

In conclusion, in this randomized pilot study comparing RE and HAE for liver metastatic SI-NET, all metastases decreased in size after 3 months, but the decrease was significantly larger in patients treated with HAE. However, no significant difference in RECIST response between groups remained after 6 months, suggesting a delayed anatomical response after treatment with RE. Although lower pretreatment $\mathrm{ADC}_{(120-800)}$ and larger increase in $\mathrm{ADC}_{(0-800)}$ at 1 month were related to improved response (decrease in tumor diameter), at 3 and 6 months respectively, no DWI-MRI threshold for the prediction of response according to RECIST could be defined. These preliminary findings indicate that HAE remains a safe option for treatment of liver metastases from SI-NET, and further studies are needed to establish the role of RE and the predictive value of DWI-MRI in these tumors.

Acknowledgements ${ }^{90} \mathrm{Y}$ resin microspheres (SIR-spheres ${ }^{\mathrm{TM}}$ ) were generously supplied by Sirtex. Professor Peter Bernhardt, Department of Radiation Physics at Sahlgrenska University Hospital is greatly appreciated for his contribution during the planning and performance of this study including responsibility of the dosimetry.

\section{Compliance with ethical standards}

Conflict of interest The authors declare that they have no conflict of interest.

Open Access This article is distributed under the terms of the Creative Commons Attribution 4.0 International License (http://crea tivecommons.org/licenses/by/4.0/), which permits unrestricted use, distribution, and reproduction in any medium, provided you give appropriate credit to the original author(s) and the source, provide a link to the Creative Commons license, and indicate if changes were made.

\section{References}

1. Ahlman H, Westeberg G, Wängberg B et al (1996) Treatment of liver metastases of carcinoid tumors. World J Surg 20:196-202

2. Swärd C, Johanson V, Nieveen van Dijkum E et al (2009) Prolonged survival after hepatic artery embolization in patients with midgut carcinoid syndrome. Br J Surg 96:517-521

3. Rajekar H, Bogammana K, Stubbs RS (2011) Selective internal radiation therapy for gastrointestinal neuroendocrine tumour liver metastases: a new and effective modality for treatment. Int J Hepatol. https://doi.org/10.4061/2011/404916

4. Kennedy AS, Dezarn WA, McNeillie P et al (2008) Radioembolization for unresectable neuroendocrine hepatic metastases using resin ${ }^{90} \mathrm{Y}$-microspheres: early results in 148 patients. Am J Clin Oncol 31:271-279

5. King J, Quinn R, Glenn DM et al (2008) Radioembolization with selective internal radiation microspheres for neuroendocrine liver metastases. Cancer 113:921-929

6. Rhee TK, Lewandowski RJ, Liu DM et al (2008) ${ }^{90} \mathrm{Y}$ Radioembolization for metastatic neuroendocrine liver tumors: preliminary results from a multi-institutional experience. Ann Surg 247:1029-1035

7. Jia Z, Paz-Fumagalli R, Frey G et al (2017) Single-institution experience of radioembolization with yttrium- 90 microspheres for unresectable metastatic neuroendocrine liver tumors. J Gastroenterol Hepatol 32:1617-1623

8. Memon K, Lewandowski RJ, Mulcahy MF et al (2012) Radioembolization for neuroendocrine liver metastases: safety, imaging, and long-term outcomes. Int J Radiat Oncol Biol Phys 83:887-894

9. Barbier CE, Garske-Román U, Sandström M et al (2016) Selective internal radiation therapy in patients with progressive neuroendocrine liver metastases. Eur J Nucl Med Mol Imaging 43:425-1431

10. Therasse P, Arbuck SG, Eisenhauer EA et al (2000) New guidelines to evaluate the response to treatment in solid tumors: European Organization for Research and Treatment of Cancer, National Cancer Institute of the United States, National Cancer Institute of Canada. J Natl Cancer Inst 92:205-216 
11. Eisenhauer EA, Therasse P, Bogaerts J et al (2009) New response evaluation criteria in solid tumours: revised RECIST guideline (version 1.1). Eur J Cancer 45:228-247

12. Padhani AR, Liu G, Mu-Koh D et al (2009) Diffusion-weighted magnetic resonance imaging as a cancer biomarker: consensus and recommendations. Neoplasia 11:102-125

13. Thoeny HC, Ross BD (2010) Predicting and monitoring cancer treatment response with DW-MRI. J Magn Reson Imaging 32:2-16

14. Bernardini M, Smadja C, Faraggi M et al (2014) Liver selective internal radiation therapy with ${ }^{90} \mathrm{Y}$ resin microspheres: comparison between pre-treatment activity calculation methods. Phys Med 30:752-764

15. Strosberg J, El-Haddad G, Wolin E et al (2017) Phase 3 Trial of ${ }^{177} \mathrm{Lu}$-Dotatate for midgut neuroendocrine tumors. N Engl J Med 376:125-135

16. Hamiditabar M, Ali M, Bolek L (2017) Safety and effectiveness of ${ }^{177}$ Lu-DOTATATE peptide receptor radionuclide therapy after regional hepatic embolization in patients with somatostatin-expressing neuroendocrine tumors. Clin Nucl Med 42:822-828

17. Luersen GF, Wei W, Tamm EP et al (2016) Evaluation of magnetic resonance (MR) biomarkers for assessment of response with response evaluation criteria in solid tumors: comparison of the measurements of neuroendocrine tumor liver metastases (NETLM) with various MR sequences and at multiple phases of contrast administration. J Comput Assist Tomogr 40:717-722

18. Bhosale P, Kwek JW, Iyer R et al (2011) Follow-up of known carcinoid liver metastases: is respiratory-gated $T_{2}$ fast spin-echo enough? Neuroendocrinology 93:241-248

19. Paprottka PM, Hoffmann RT, Haug A et al (2012) Radioembolization of symptomatic, unresectable neuroendocrine hepatic metastases using yttrium-90 microspheres. Cardiovasc Interv Radiol 35:334-342

20. Cao CQ, Yan TD, Bester L et al (2010) Radioembolization with yttrium microspheres for neuroendocrine tumour liver metastases. Br J Surg 297:537-543

21. Peker A, Cicek O, Soydal C et al (2015) Radioembolization with yttrium-90 resin microspheres for neuroendocrine tumor liver metastases. Diagn Interv Radiol 21:54-59

22. Saxena A, Chua TC, Bester L et al (2010) Factors predicting response and survival after yttrium-90 radioembolization of unresectable neuroendocrine tumor liver metastases: a critical appraisal of 48 cases. Ann Surg 251:910-916

23. Fidelman N, Kerlan RK Jr, Hawkins RA et al (2016) Radioembolization with ${ }^{90} \mathrm{Y}$ glass microspheres for the treatment of unresectable metastatic liver disease from chemotherapy-refractory gastrointestinal cancers: final report of a prospective pilot study. J Gastrointest Oncol 7:860-874

24. Deng J, Rhee TK, Sato KT et al (2006) In vivo diffusionweighted imaging of liver tumor necrosis in the VX2 rabbit model at 1.5 Tesla. Invest Radiol 41:410-414

25. Herneth AM, Guccione S, Bednarski M (2003) Apparent diffusion coefficient: a quantitative parameter for in vivo tumor characterization. Eur J Radiol 45:208-213

26. Kukuk GM, Murtz P, Traber F et al (2013) Diffusion-weighted imaging with acquisition of three $b$-values for response evaluation of neuroendocrine liver metastases undergoing selective internal radiotherapy. Eur Radiol 24:267-276

27. Cui Y, Zhang XP, Sun YS et al (2008) Apparent diffusion coefficient: potential imaging biomarker for prediction and early detection of response to chemotherapy in hepatic metastases. Radiology 248:894-900

28. Koh DM, Scurr E, Collins D et al (2007) Predicting response of colorectal hepatic metastasis: value of pretreatment apparent diffusion coefficients. AJR Am J Roentgenol 188:1001-1008

29. Dudeck O, Zeile M, Wybranski C et al (2010) Early prediction of anticancer effects with diffusion-weighted MR imaging in patients with colorectal liver metastases following selective internal radiotherapy. Eur Radiol 20:2699-2706

30. Atassi B, Bangash AK, Bahrani A et al (2008) Multimodality imaging following ${ }^{90} \mathrm{Y}$ radioembolization: a comprehensive review and pictorial essay. Radiographics 28:81-99

31. Pieper CC, Sprinkart AM, Meyer C et al (2016) Evaluation of a simplified intravoxel incoherent motion (IVIM) analysis of diffusion-weighted imaging for prediction of tumor size changes and imaging response in breast cancer liver metastases undergoing radioembolization: a retrospective single center analysis. Medicine 95(e3275):1-9

32. Lambregts DMJ, Beets GL, Maas M et al (2011) Tumour ADC measurements in rectal cancer: effect of ROI methods on ADC values and interobserver variability. Eur Radiol 21:2567-2574 